\title{
Paradoxical Cerebral Air Embolism Occurred with Postural Change during Rehabilitation, in a Patient with Ipsilateral Internal Carotid Artery Occlusion
}

\author{
Kentaro Suzuki, Masayuki Ueda, Arata Abe, Yasuhiro Nishiyama, Seiji Okubo, \\ Kenichiro Katsura and Yasuo Katayama
}

\begin{abstract}
We report a 76-year-old man with paradoxical cerebral air embolism. He developed consciousness disturbance and left hemiparesis after a postural change in rehabilitation. CT showed multiple air densities within the right hemisphere. An echocardiography showed a large right-to-left (RL) shunt. We considered the reason to be that a small amount of air entered, and the Valsalva-like maneuver with the postural change moved air into arterial circulation through the RL shunt and embolized a brain artery. The present case showed that even a small amount of air in the venous circulation may become a potential risk for cerebral air embolism, especially in the presence of a large RL shunt.
\end{abstract}

Key words: stroke, air embolism, infarction, RL shunt

(Intern Med 51: 1107-1109, 2012)

(DOI: 10.2169/internalmedicine.51.7032)

\section{Introduction}

Cerebral air embolism is a serious complication of various conditions that cause direct entry or accumulation of air in the arteries, such as trauma, diving, surgery, and lung biopsy (1). Air that enters the veins does not usually result in cerebral air embolism, but paradoxical cerebral air embolism can rarely occur in persons with a right-to-left (RL) shunt. We encountered a male patient with paradoxical cerebral air embolism that was probably due to a small volume of air inflow through a central venous line. Cranial computed tomography (CT) showed multiple air densities within the territory of the right middle cerebral artery (MCA) despite the presence of asymptomatic occlusion of the ipsilateral internal carotid artery (ICA). Here, we discuss the distribution of cerebral air densities and other unusual features of paradoxical cerebral air embolism associated with a small influx of intravenous air.

\section{Case Report}

A 76-year-old man with triple-vessel coronary artery disease was admitted to our hospital for coronary artery bypass grafting. His risk factors for atherosclerosis were hypertension, dyslipidemia, diabetes mellitus, and cigarette smoking. Asymptomatic right internal carotid artery occlusion had been noted by magnetic resonance angiography (MRA). Offpump bypass surgery was performed without any complications. At 2 days after surgery, he suddenly developed disturbance of consciousness with left hemiparesis, just after he had been moved into a wheelchair for rehabilitation. His blood pressure was $132 / 80 \mathrm{mmHg}$ and there was no significant difference observed between supine and sitting position. The National Institute of Health Stroke Scale (NIHSS) score was 30 at his initial assessment, and he was transferred to our stroke care unit. Initial brain CT (at 20 minutes after the onset) showed multiple air densities within the right middle cerebral artery territory, while no such air densities were observed in the posterior territory (Fig. 1). These air densities were markedly diminished when head CT was repeated 6 


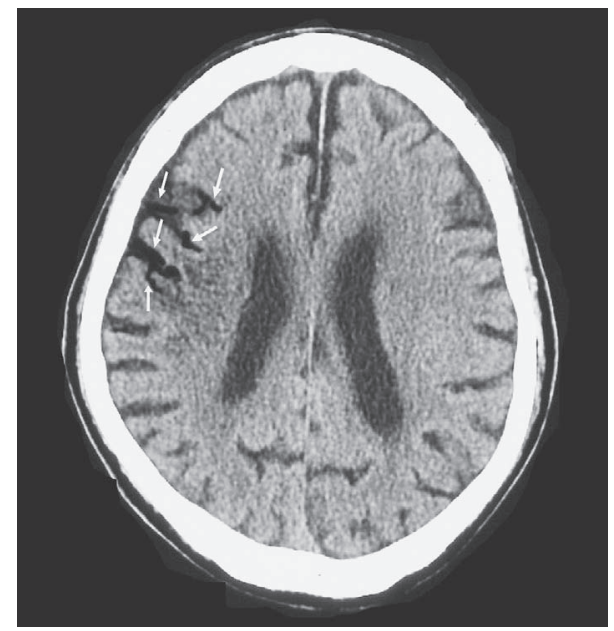

Figure 1. Initial head CT at 20 min after symptom onset shows multiple air densities (white arrow) within the right middle cerebral artery (MCA) territory, but not in the left hemisphere and the posterior circulation territory.

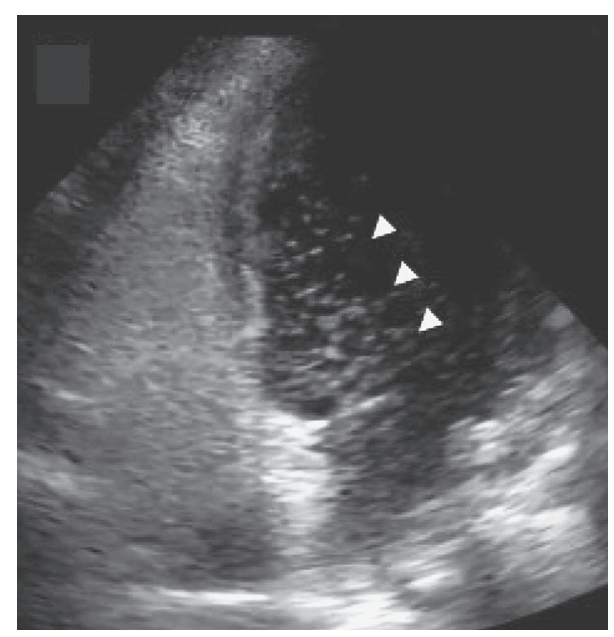

Figure 2. Transthoracic echocardiography using contrast echo method displays an influx of a large number of microbubbles into the left atrium (white arrow head) immediately after injection of saline containing microbubbles.

hours later. We performed lower extremity venous ultrasound and we could not find any venous thrombosis before the onset of air embolism. Transthoracic echocardiography with the contrast echo method revealed massive influx of microbubbles into the left atrium immediately after the injection of saline containing microbubbles, indicating that he had a large intracardiac RL shunt (Fig. 2). A central venous line in the right internal jugular vein was the only intravascular catheter at the onset of symptoms. On day 12, acute cerebral infarctions in the right MCA territory were observed by diffusion weighted image and fluid attenuation inversion recovery image (Flair) of magnetic resonance imaging (MRI) (Fig. 3). MRA showed occluded right internal carotid artery (ICA) and collateral vascular signals (Fig. 4). We administered glycerol (2) and free radical scavenger, edaravone (3), for the treatment of the cerebral infarction.

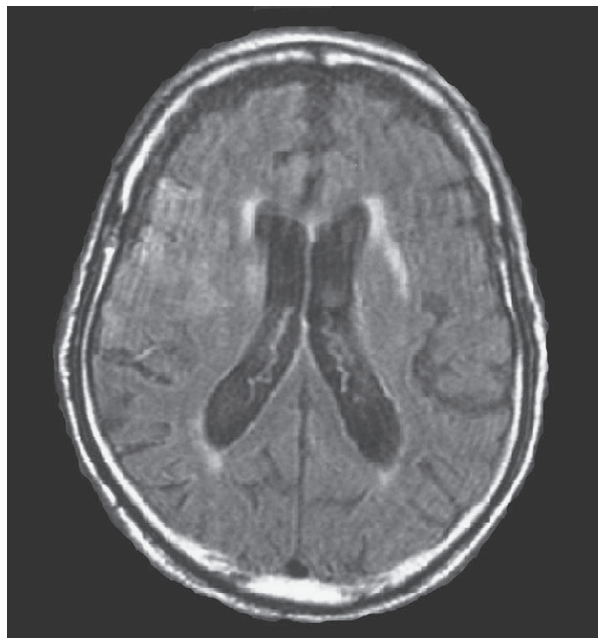

Figure 3. Fluid attenuation inversion recovery image (Flair) of MRI on day 12.

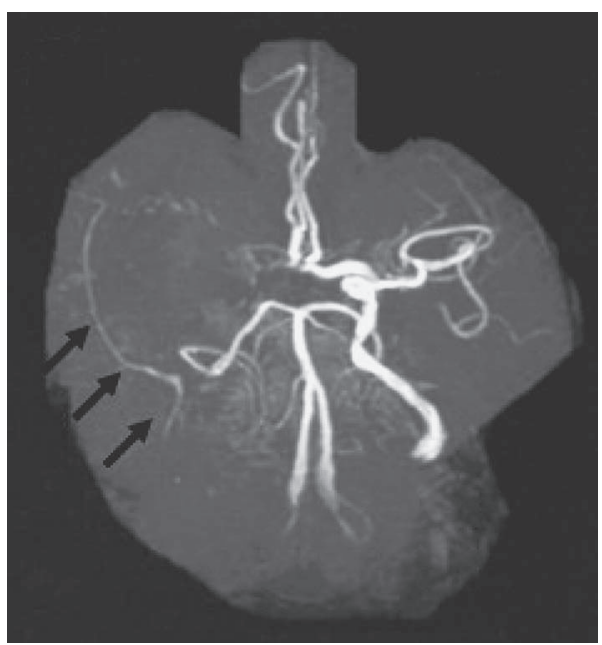

Figure 4. MRA showed occluded right internal carotid artery (ICA).

His neurological symptoms gradually improved, and the NIHSS score was 10 on day 20 and he was moved to the rehabilitation hospital.

\section{Discussion}

The present patient had a large intracardiac RL shunt on transthoracic echocardiography (contrast echo method). A patent foramen ovale is a common finding with a mean prevalence of $26 \%$ in autopsy studies (4). Detection of numerous microbubbles in the left atrium immediately after the injection of saline containing microbubbles in the present patient suggested a large patent foramen ovale, based on the criterion (inflow of more than 30 microbubbles into the left atrium) reported previously (5). Since more than two days passed after cardiac operation, and since the central venous line was the only intravascular catheter at symptom onset, an influx of a small volume of air through this line combined with the large RL shunt and a valsalva-like maneuver when standing and moving into the wheel-chair, might have 
been the mechanism of the paradoxical cerebral air embolism in the present patient.

Griese et al. investigated 39 patients with cerebral air embolism following cardiac surgery. They found that 38 out of 39 patients had air embolism predominantly affecting the right hemisphere, while one patient displayed only left hemispheric involvement (6). They suspected one of the reasons of the difference in prevalence between the two hemispheres may have been from the anatomical setting of the brachiocephalic artery, which is the first major branch of the aortic arch. The present patient had multiple air densities in the right MCA territory on initial CT scans, despite occlusion of the ipsilateral ICA. Since air densities were not observed in the left hemisphere or the posterior territory, there is a possibility that the inflow of air into the brain might occur through a collateral route of the right external carotid artery-superficial temporal vessel-peripheral middle cerebral artery. This case may indicate that even a patient with right internal carotid artery occlusion may have a cerebral air embolism in the right hemisphere, suggesting that it is important to consider the likelihood of air embolism when abnormally low densities are seen in the right hemisphere with very acute CT scan.

When air embolism was suspected, the head-down position (7) and the left lateral position (8) have been recommended in order to collect the gaseous mass in the tip of the right ventricle so as to free the pulmonary arterial tract. As a treatment of air embolism, hyperbaric oxygen is recommended (9-12). In the present case, hyperbaric oxygen treatment was not possible because the patient was in such a severe state and intubated with a ventilator. Thus we tried the usual treatment for cerebral infarction, and the treatment seemed to be successful.

Intravenous hyperalimentation is a useful tool for patients with serious conditions, but small volumes of air may enter the venous system along with drug injection in the routine clinical setting. Complications have been reported with as little as $20 \mathrm{ml}$ of air (13), and 200-300 $\mathrm{ml}$ of air was lethal (14). In the present case, before air embolism occurred, several catheters connected to the central venous catheter had been changed. So we think via intra-catheter a small amount of air entered when connected catheters were changed, and the air entered through the central venous catheter. Usually such a small amount of air does not lead to serious complications, however, if the air directly enters into the cerebral circulation, it is reported that $2-3 \mathrm{ml}$ of air can be fatal (15). The present case clearly emphasizes the potential risk of cerebral air embolism due to a small amount of venous air entering in patients with a large RL shunt.

The authors state that they have no Conflict of Interest (COI).

\section{References}

1. Shaikh N, Ummunisa F. Acute management of vascular air embolism. J Emerg Trauma Shock 2: 180-185, 2009.

2. Bayer AJ, Pathy MS, Newcombe R. Double-blind randomised trial of intravenous glycerol in acute stroke. Lancet 1: 405-408, 1987.

3. Shinohara Y, Saito I, Kobayashi S, Uchiyama S. Edaravone (radical scavenger) versus sodium ozagrel (antiplatelet agent) in acute noncardioembolic ischemic stroke (EDO trial). Cerebrovasc Dis 27: 485-492, 2009.

4. Homma S, Sacco RL. Patent foramen ovale and stroke. Circulation 112: 1063-1072, 2005.

5. Kobayashi K, Iguchi Y, Kimura K, et al. Contrast transcranial Doppler can diagnose large patent foramen ovale. Cerebrovasc Dis 27: 230-234, 2009.

6. Griese H, Seifert D, Koerfer R. Cortical infarction following cardiosurgical procedures - air embolism as a probable cause. Eur Neurol 61: 343-349, 2009.

7. Durant TM, Long J, Oppenheimer MJ. Pulmonary (venous) air embolism. Am Heart J 33: 269-281, 1947.

8. Rodriguez RA, Cornel G, Weerasena NA, Pham B, Splinter WM. Effect of Trendelenburg head position during cardiac deairing on cerebral microemboli in children: a randomized controlled trial. $\mathbf{J}$ Thorac Cardiovasc Surg 121: 3-9, 2001.

9. Bacha S, Annane D, Gajdos P. Iatrogenic air embolism. Presse Med 25: 1466-1472, 1996 (in French).

10. Dutka AJ. A review of the pathophysiology and potential application of experimental therapies for cerebral ischemia to the treatment of cerebral arterial gas embolism. Undersea Biomed Res 12: 403-421, 1985.

11. Kindwall EP. Massive surgical air embolism treated with brief recompression to six atmospheres followed by hyperbaric oxygen. Aerosp Med 44: 663-666, 1973.

12. McDermott JJ, Dutka AJ, Koller WA, Pearson RR, Flynn ET. Comparison of two recompression profiles in treating experimental cerebral air embolism. Undersea Biomed Res 19: 171-185, 1992.

13. Natal BL, Dotty CI. Venous air embolism. Medscape 2009. http:// emedicine.medscape.com/article/761367-overview

14. Mirski MA, Lele AV, Fitzsimmons L, Toung TJ. Diagnosis and treatment of vascular air embolism. Anesthesiology 106: 164-177, 2007.

15. Ho AM. Is emergency thoracotomy always the most appropriate immediate intervention for systemic air embolism after lung trauma? Chest 166: 234-237, 1999.

(C) 2012 The Japanese Society of Internal Medicine

http://www.naika.or.jp/imindex.html 